\title{
DYNAMIQUE DES PEUPLEMENTS DE POISSONS DANS LE RÉSERVOIR DE PETIT-SAUT (FLEUVE SINNAMARY, GUYANE FRANÇAISE).
}

\section{B. DE MÉRONA}

IRD, Institut de Recherche pour le Développement, Centre de Cayenne, B.P. 165 97323 CAYENNE Cedex, France, e-mail : merona@ cayenne.ird.fr

\section{RÉSUMÉ}

La modélisation des effets des barrages hydroélectriques nécessite un grand nombre de données d'observation dans chacune des situations concrètes. En zone tropicale ces données sont souvent incomplètes et de nature hétérogène rendant difficile la comparaison entre des situations différentes. En janvier 1994, Electricité de France (EDF : Compagnie Nationale française d'Electricité) a procédé à la fermeture du barrage de Petit-Saut sur le fleuve Sinnamary en Guyane française. A cette occasion, un suivi environnemental a été mené sur une période s'étendant depuis 5 années avant la fermeture jusqu'à 7 années après. En ce qui concerne les poissons, trois phases successives dans le processus de colonisation du lac ont été identifiées. Pendant la phase de mise en eau, les captures de poissons ont rapidement augmenté alors que la richesse spécifique diminuait. Une espèce dominait le peuplement. Durant les 2 années qui ont suivi, les captures se sont maintenues à un niveau élevé alors que la richesse spécifique continuait à diminuer. Le peuplement était dominé par deux espèces capables de tirer avantage des nouvelles ressources offertes par le lac. Enfin, au cours des 4 années suivantes, les captures diminuaient lentement et régulièrement et la composition spécifique se modifiait profondément. Ce schéma semble conforme à ce qui a été observé dans plusieurs grandes retenues en zone tropicale.

Mots-clés : Amérique du Sud, barrage, poisson, communauté, densité, richesse spécifique, structure, composition spécifique.

\section{FIRST COMMUNITY DYNAMICS IN THE PETIT-SAUT RESERVOIR (SINNAMARY RIVER, FRENCH GUIANA).}

\begin{abstract}
A large amount of descriptive data of concrete cases is needed in order to establish models of environmental effects of dams. In the tropics these data are often incomplete or of different types and the comparison between situations is difficult. In January 1994 EDF (French State Electricity Company) closed Petit-Saut dam on the Sinnamary River in French Guiana. On this occasion an ecological monitoring program was conducted
\end{abstract}


covering the period 1990-2000. Regarding fish, 3 phases could be recognized in the process of colonization of the new man-made lake. During the filling phase, captures grew rapidly when species richness decreased. One species dominated the community. In the 2 following years, captures remained high and species richness was decreasing even more. Community was dominated by two species able to take advantage from the new food resources available in the environment. At last, during the 4 following years, captures decreased slowly and regularly when the species composition was changing drastically. This picture seems to be in accordance with the observations in other large man-made lakes in the tropics.

Key-words : South America, dam, fish, community, density, species richness, structure, species composition.

\section{INTRODUCTION}

En janvier 1994, Electricité de France (EDF : Compagnie française d'Electricité) fermait le barrage de Petit-Saut sur le fleuve Sinnamary en Guyane française. II s'agissait du premier ouvrage construit par cette compagnie en zone tropicale mais non pas du premier dans la région. En effet, depuis les années 60 , un certain nombre de barrages ont été implantés sur des fleuves et rivières de la grande région amazonienne. Dès 1964, le barrage de Brokopondo au Surinam, pays voisin de la Guyane française, a inondé près de $1500 \mathrm{~km}^{2}$ de forêt primaire avec des conséquences dramatiques sur la qualité de l'eau (VAN DER HEIDE, 1982). Au Brésil deux petits barrages ont été implantés en Amazonie dans les années 70 : Paredão dans l'Etat d'Amapá avec une retenue d'environ $23 \mathrm{~km}^{2}$ et Curuá-Una dans l'État du Pará dont la retenue s'étend sur $78 \mathrm{~km}^{2}$. A partir des années 80 se développent dans ce pays des projets de grands barrages dont les plus importants en Amazonie sont Tucurui et Balbina qui ont formé des retenues de plus de $2000 \mathrm{~km}^{2}$.

L'implantation de ces ouvrages a naturellement des conséquences drastiques sur l'environnement aquatique et depuis de nombreuses années la recherche scientifique tente de comprendre les mécanismes qui président aux changements observés (LOWE-MCCONNELL, 1966 ; OBENG, 1969 ; ACKERMANN et al., 1973 ; BALON et COCHE, 1974 ; KAPETSKY et PETR, 1984 ; STRASKRABA et al., 1993 ; TUNDISI et STRASKRABA, 1999). Cependant l'établissement de modèles permettant de prévoir les effets des barrages, et donc d'en minimiser les conséquences négatives, se heurte à un grand nombre de difficultés. En premier lieu les conditions climatiques, physico-chimiques et biologiques des rivières concernées sont très variées et celles-ci réagissent donc de manière très diversifiée au stress produit par la transformation d'une partie du cours en lac (THORNTON et RAST, 1993 ; LIND et al., 1993). Ainsi de nombreux paramètres doivent être considérés dans les modèles et un grand nombre de données d'observation sont nécessaires pour les ajuster. Une autre difficulté tient justement à la relative rareté ou à l'indisponibilité des observations. Le plus souvent la mise en place des ouvrages s'est accompagnée d'études sur les conséquences environnementales, mais la plupart du temps ces études sont incomplètes. Dans certains cas on ne dispose pas de données avant barrage, dans d'autres les observations sont interrompues avant la stabilisation du nouveau système. De plus, beaucoup de données générées par ces études restent confidentielles, sous forme de rapports inaccessibles. II y a enfin un problème lié à l'hétérogénéité des données et en particulier celles qui ont trait au compartiment biologique de l'écosystème transformé. Le plan d'échantillonnage, le degré de connaissance systématique de la faune et de la flore, sont autant de facteurs qui limitent la portée des comparaisons entre systèmes.

Il est donc essentiel de multiplier les observations portant sur de nouveaux barrages et c'est l'objectif de ce travail. 


\section{MATÉRIEL ET MÉTHODES}

\section{Site d'étude}

Le fleuve Sinnamary, situé au centre de la Guyane française, a une extension de $250 \mathrm{~km}$ et un bassin versant de $6565 \mathrm{~km}^{2}$ (Figure 1). II coule du sud au nord le long d'un profil longitudinal décrit par TITO DE MORAIS et LAUZANNE, 1994. Ces auteurs distinguent 5 zones : 1) la tête du bassin qui s'étend sur moins de $50 \mathrm{~km}$ et qui présente une forte pente entrecoupée de sauts peu accentués, 2) une zone amont de près de $100 \mathrm{~km}$, de pente généralement faible, formée de biefs d'une dizaine de $\mathrm{km}$ et de sauts peu marqués, 3) une zone centrale d'une cinquantaine de $\mathrm{km}$ avec une pente forte et entrecoupée de sauts à dénivelé important, 4) une zone basse de pente très faible, formée de longs biefs et de quelques rapides peu marqués, et enfin 5) l'estuaire qui s'étend sur quelques $\mathrm{km}$ et qui correspond à la zone de pénétration du coin salé*. La quasi-totalité du cours est bordée de forêt primaire.
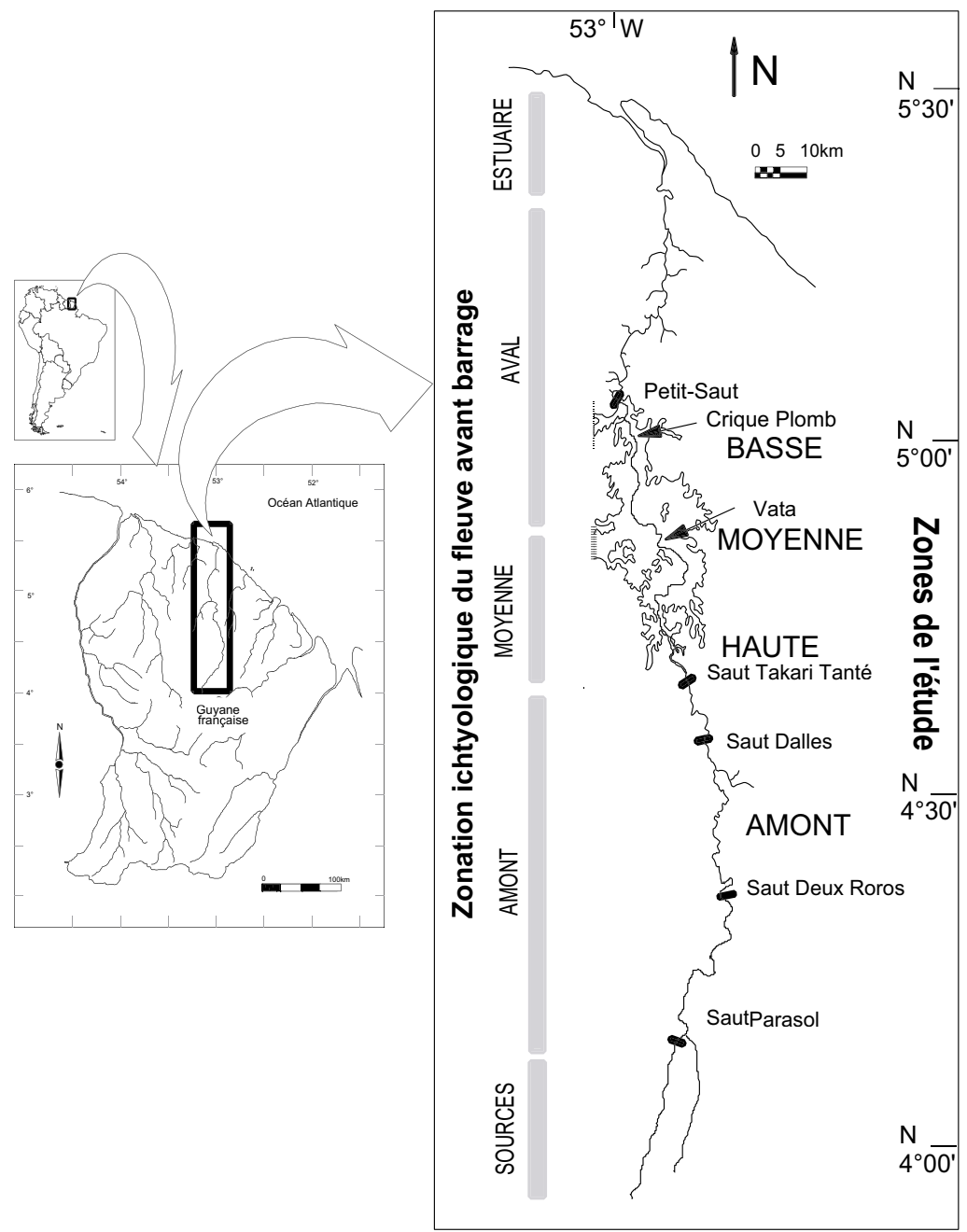

\section{Figure 1}

Localisation de la zone d'étude et des stations d'échantillonnage.

\section{Figure 1}

Geographical location of study site and sampling stations.

\footnotetext{
* intrusion d'eau de mer profonde dans les estuaires des rivières.
} 
Le régime hydrologique, caractérisé par l'alternance de crues, entre décembre et juillet, et d'étiages d'août à novembre, est marqué par de rapides et importantes variations journalières et par d'amples variations inter-annuelles (Figure 2). Le débit moyen est d'environ $300 \mathrm{~m}^{3} \cdot \mathrm{s}^{-1}$.

Le barrage de Petit-Saut fut érigé au niveau du premier saut en remontant la rivière, situé à quelques $60 \mathrm{~km}$ de l'estuaire. C'est un ouvrage en béton d'une hauteur de $45 \mathrm{~m}$, comportant 4 turbines d'une capacité de $100 \mathrm{~m}^{3} . \mathrm{s}^{-1}$ chacune, un déversoir de surface et un évacuateur de fond. La mise en eau de la retenue a débuté en janvier 1994 et s'est poursuivie jusqu'en mai 1995, lorsque le niveau d'eau dans le réservoir a atteint sa valeur maximum de $35 \mathrm{~m}$ (Figure 2). A cette cote, le réservoir couvre une surface d'environ $360 \mathrm{~km}^{2}$ et a une extension longitudinale d'environ $80 \mathrm{~km}$. Son pourtour est très dendritique et il est parsemé d'une grande quantité d'îles de taille variée. La totalité de la zone inondée était recouverte de forêt primaire et les premiers mois de l'inondation ont été marqués par une désoxygénation importante de l'eau du fait de la décomposition du matériel végétal au fond (RICHARD et al., 1997).

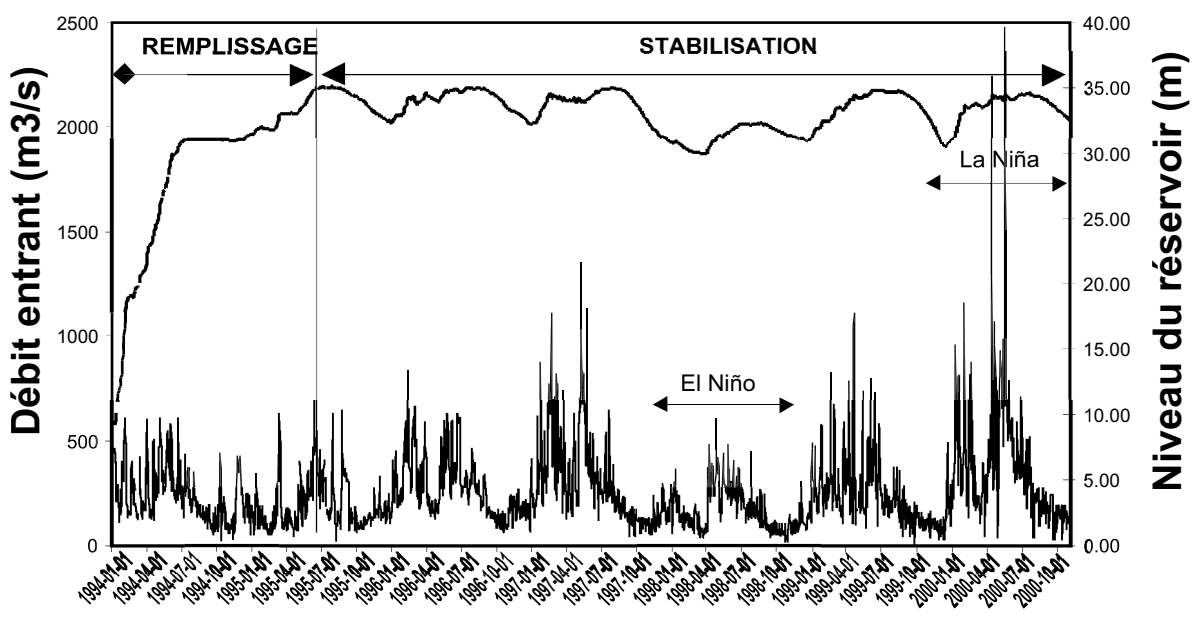

Figure 2

Hydrogramme du Sinnamary à l'amont de la retenue et évolution du niveau d'eau dans la retenue entre 1994 et 2000.

\section{Figure 2}

Hydrogram of Sinnamary River upstream from the reservoir and variation of water level in the reservoir between 1994 and 2000.

\section{Echantillonnage}

Trois zones ont été échantillonnées : la première, mobile, située au niveau du front d'inondation $(\mathrm{H})$, la deuxième localisée dans la partie médiane du lac au niveau de l'ancien saut Vata (M) et la troisième dans la partie basse au niveau de la crique Plomb (B) (Figure 1). Dans les deux dernières zones, deux types de milieux ont été prospectés : les eaux libres (EL) et les milieux fermés $(F)$. Au début de l'inondation, seuls des milieux fermés proches de l'ancien cours du fleuve étaient accessibles du fait de l'épaisseur de la végétation dans la canopée de la forêt ennoyée, mais, à mesure que la végétation mourait, l'accès à des zones plus profondes devenait possible.

Pendant les trois années qui ont suivi la fermeture du barrage, ces stations ont été échantillonnées mensuellement, puis durant les quatre années suivantes, les collectes ont été semestrielles. Pour le passage du suivi mensuel au suivi semestriel, nous nous sommes basés sur une analyse de la meilleure approximation de la capture moyenne 
annuelle durant les trois années d'observations mensuelles à partir de deux collectes dans l'année. Cette analyse a permis de positionner les collectes semestrielles en juin et en novembre. Ce sont d'ailleurs deux périodes clés dans le cycle hydrologique : la première correspond au maximum de la crue et la deuxième à la fin de l'étiage.

Parallèlement aux pêches dans la retenue, des collectes ont été effectuées dans la partie du fleuve à l'amont de la retenue. Celles-ci ont débuté dans une station proche du lac en janvier 1996 (à l'amont de saut Takari Tanté) et se sont poursuivies à partir de novembre 1996 dans trois stations situées à des distances croissantes de la retenue : saut Takari Tanté, saut Dalles et saut Deux Roros (Figure 1).

L'échantillonnage des poissons a été effectué à l'aide de filets maillants. On a utilisé une ou deux batteries de 10 filets de maille croissante entre $10 \mathrm{~mm}$ et $70 \mathrm{~mm}$ entre nœuds, de $25 \mathrm{~m}$ de longueur et $2 \mathrm{~m}$ de chute dans l'eau. Les filets ont été posés avant la tombée de la nuit à partir de $17 \mathrm{~h}$ et relevés le matin après le lever du jour à partir de $7 \mathrm{~h}$.

Les captures de chacune des mailles ont été séparées et sur le terrain, les poissons ont été individuellement identifiés à l'espèce, mesurés au $\mathrm{mm}$ près et pesés au dixième de gramme à l'aide d'une balance digitale SARTORIUS QS4000. Lorsque le nombre de poissons capturés était très élevé, un certain nombre de spécimens de la même espèce dans une maille donnée était pesé en groupe afin d'accélérer le traitement et éviter un pourrissement généralisé de la prise.

\section{Traitement des données}

Les échantillons sont caractérisés par trois indices :

- un indice d'abondance exprimé par la capture en nombre de spécimens par unité d'effort soit une nuit de pêche et une batterie de filets (NOMBRE).

- La diversité calculée selon la formule de Shannon :

$$
\mathrm{I}=-\Sigma \mathrm{p}_{\mathrm{i}} \cdot \log _{2} \mathrm{p}_{\mathrm{i}} \quad \text { (avec } \mathrm{p}_{\mathrm{i}}=\text { fréquences relatives des espèces) }
$$

- La régularité calculée selon la formule de l'équitabilité :

$$
E=I / \log _{2} R \quad \text { (avec } R=\text { nombre d'espèces dans l'échantillon) }
$$

En vue de tester l'évolution temporelle (facteur ANNEE) et l'effet de la localisation de l'échantillon (facteur POSITION) sur les différents paramètres des échantillons (capture, diversité et équitabilité), nous avons utilisé l'analyse de variance à deux facteurs que nous avons appliqué aux seuls prélèvements de juin et de novembre. Les échantillons sont comparés deux à deux par des tests $t$ de Student et les probabilités associées sont corrigées par la méthode de Bonferroni : le niveau de signification est divisé par le nombre de comparaisons. Le logiciel utilisé est Systat ${ }^{\circledR}$ v.9.

L'évolution de la richesse spécifique a été évaluée par la valeur asymptotique d'une courbe logistique ajustée au nombre cumulé d'espèces dans les échantillons successifs au cours de chacune des périodes 1994, 1995, 1996 et l'intervalle 1997 à 2000 . L'équation de la courbe logistique est de la forme:

$$
R=\operatorname{Rmax}^{*}\left(1-\mathrm{e}^{-k(x-c)}\right)
$$

avec $\mathrm{R}$ : nombre d'espèces ; Rmax : nombre asymptotique d'espèces (richesse estimée de l'assemblage) ; $x$ : nombre d'échantillons successifs ; $k, c$ : paramètres de l'équation. 
L'ajustement est obtenu par la méthode des moindres carrés en utilisant le logiciel Systat $^{\oplus}$ v.9.

Le suivi de la composition spécifique des échantillons a été fait par une analyse des correspondances à l'aide du logiciel ADE-4 (THIOULOUSE et al., 1997).

\section{RÉSULTATS}

\section{Capture}

Dès les premiers mois de l'inondation les captures ont augmenté rapidement (Figure 3). Elles sont restées à un niveau élevé pendant les trois premières années, culminant dans certaines stations jusqu'à plus de 1200 poissons par batterie et par nuit. Elles étaient, pendant cette période, largement supérieures aux captures réalisées dans le fleuve en amont, les plus fortes étant réalisées au niveau des zones médiane et haute du lac. La variabilité de la capture dans la retenue était importante. Après 1996, les captures ont commencé à décliner et ce déclin s'est poursuivi jusqu'à la fin des observations. Une analyse de variance portant sur les seuls prélèvements de juin et de novembre montre que les facteurs ANNEE et POSITION ont un effet hautement significatif sur la capture (Tableau I et Figure $4 a$ et b). On observe, de plus, une interaction significative entre ces deux paramètres. Les probabilités associées aux comparaisons deux à deux corrigées par la méthode de Bonferroni ne montrent des différences significatives entre années que pour les zones haute et moyenne de la retenue (Tableau II).

La répartition des captures n'était pas homogène. Elles étaient plus importantes dans les zones haute et moyenne que dans la zone basse et que dans la rivière en amont (Tableau I et Figure 4b). En revanche il n'y avait pas de différence significative entre les habitats d'eau libre et les milieux fermés dans les zones où ces deux habitats ont pu être échantillonnés (Tableau III).

\section{Diversité et régularité}

La diversité était de même ordre de grandeur dans les différentes zones mais, en revanche, la régularité des échantillons était en moyenne plus faible dans la zone haute du réservoir, proche du fleuve (Tableau I et Figure 4c). Là également une interaction significative est relevée entre les deux paramètres, mais aucune comparaison deux à deux ne montre de différence significative.

Lorsque l'on ne considère que les prélèvements réalisés dans les parties moyenne et basse du lac, c'est à dire ceux où deux milieux différents ont été échantillonnés, tous les paramètres des échantillons sont influencés par le facteur année (Tableau III et Figure 5). La diversité de Shannon et l'équitabilité ne présentaient pas de tendance temporelle nette ni ne montraient de différence significative entre milieux (Tableau III et Figure $5 c$ et $d$ ).

\section{Richesse spécifique}

La richesse spécifique estimée dans la rivière à partir des prélèvements successifs réalisés entre 1997 et 2000 est de 61 espèces (Tableau IV). Dans la retenue ce paramètre est maximum en 1994 avec 67 espèces puis diminue régulièrement jusqu'en 1997 (Tableau IV et Figure 6). En 1996 la richesse cumulée n'est plus que de 43 espèces. Au cours de la période 1997 à 2000, le nombre d'espèces augmente de nouveau dans la retenue et semble encore en évolution. 


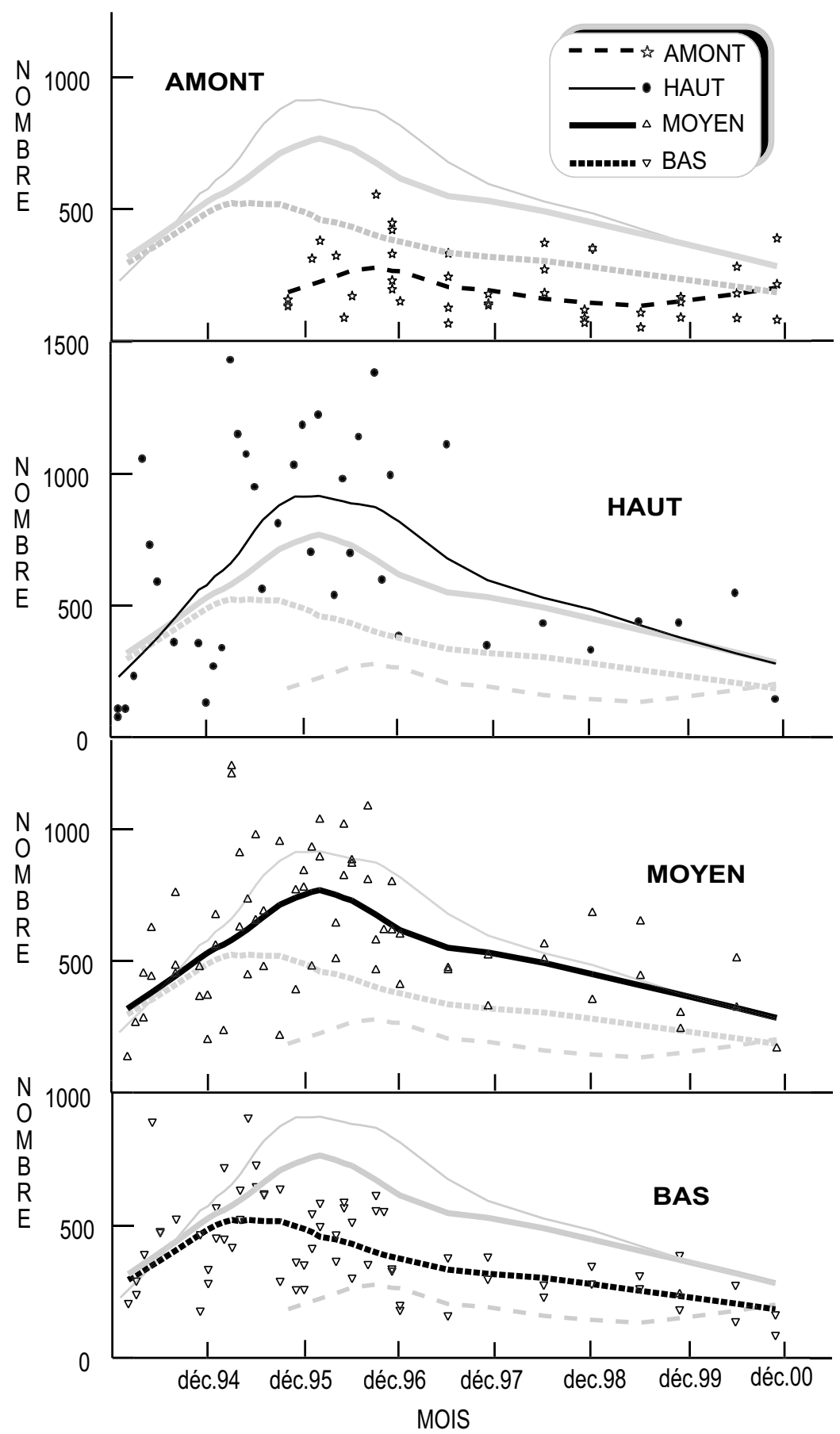

Figure 3

Evolution de la capture de poissons dans la retenue de Petit-Saut et dans le fleuve à l'amont entre janvier 1994 et novembre 2000. Les courbes représentent des fonctions de lissage LOWESS de tension 0,5 appliquées à chacune des séries.

Figure 3

Changes in fish catches in Petit-Saut reservoir and in the river upstream between January 1994 and November 2000. The curves are LOWESS smoothing functions with tension 0.5 applied to each of the series. 

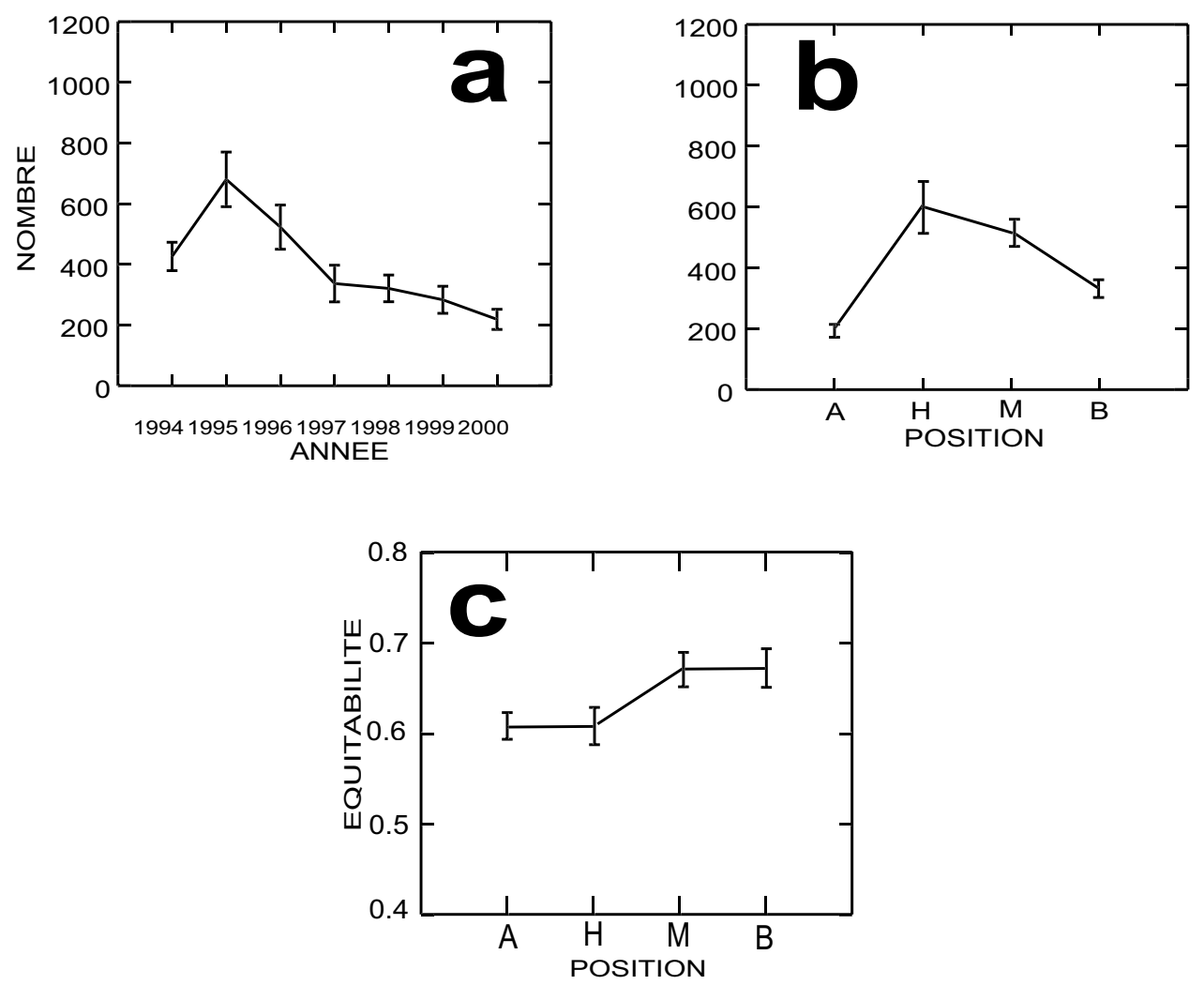

Figure 4

Variations statistiquement significatives des paramètres des échantillons en fonction des facteurs année et position. a : nombre capturé en fonction de l'année. b : nombre capturé en fonction de la position. c : équitabilité en fonction de la position. Les barres d'erreurs correspondent à une erreur type.

Figure 4

Significant changes in samples parameters with year and position. a: captured number with year. b: captured number with position. c: equitability with position. Error bars correspond to one standard error.

\section{Tableau I}

Résultats de l'Analyse de Variance à deux facteurs de variabilité (Année et Position) sur les paramètres de l'ensemble des échantillons réalisés dans le reservoir de Petit-Saut et à l'amont.

Table I

Results of two way analysis of variance with year and position on parameters of all the samples taken in Petit-Saut reservoir and in the upstream river.

\begin{tabular}{|l|c|c|c|}
\hline Facteurs & Nombre & Diversité & Equitabilité \\
\hline Année & $<0,001$ & 0,565 & 0,184 \\
\hline Position & $<0,001$ & 0,602 & 0,031 \\
\hline Année ${ }^{*}$ Position & 0,069 & 0,011 & 0,001 \\
\hline
\end{tabular}




\section{Tableau II}

Probabilités de Bonferroni associées aux comparaisons deux à deux des échantillons réalisés en juin et novembre entre 1994 et 2000 . En haut de la diagonale échantillons de l'amont et de la partie basse du réservoir ; en bas échantillons des parties haute et moyenne du réservoir.

\section{Table II}

Bonferroni probabilities associated to the pairwise comparisons of samples taken in June and November between 1994 and 2000. Above the diagonal, samples from the river upstream and the lower section of the reservoir; below the diagonal, samples from the medium and upper sections of the reservoir.

\begin{tabular}{|c|c|c|c|c|c|c|c|}
\hline & 1994 & 1995 & 1996 & 1997 & 1998 & 1999 & 2000 \\
\hline 1994 & & $\begin{array}{l}\text { A :n.e. } \\
B: 1,000\end{array}$ & $\begin{array}{l}A: 1,000 \\
B: 1,000\end{array}$ & $\begin{array}{l}A: 1,000 \\
B: 1,000\end{array}$ & $\begin{array}{l}A: 1,000 \\
B: 1,000\end{array}$ & $\begin{array}{l}A: 1,000 \\
B: 1,000\end{array}$ & $\begin{array}{l}A: 1,000 \\
B: 1,000\end{array}$ \\
\hline 1995 & $\begin{array}{l}M: 1,000 \\
H: 0,188\end{array}$ & & $\begin{array}{l}\text { A : n.e. } \\
B: 1,000\end{array}$ & $\begin{array}{l}\text { A : n.e. } \\
B: 1,000\end{array}$ & $\begin{array}{l}\text { A : n.e. } \\
B: 1,000\end{array}$ & $\begin{array}{l}\text { A : n.e. } \\
B: 1,000\end{array}$ & $\begin{array}{l}\text { A : n.e. } \\
\text { B :0.209 }\end{array}$ \\
\hline 1996 & $\begin{array}{l}M: 1,000 \\
H: 1,000\end{array}$ & $\begin{array}{l}M: 1,000 \\
H: 1,000\end{array}$ & & $\begin{array}{l}\text { A }: 1,000 \\
B: 1,000\end{array}$ & $\begin{array}{l}\text { A : } 1,000 \\
B: 1,000\end{array}$ & $\begin{array}{l}\text { A : } 1,000 \\
B: 1,000\end{array}$ & $\begin{array}{l}A: 1,000 \\
B: 1,000\end{array}$ \\
\hline 1997 & $\begin{array}{l}M: 1,000 \\
H: 1,000\end{array}$ & $\begin{array}{l}M: 1,000 \\
H: 1,000\end{array}$ & $\begin{array}{l}M: 0,370 \\
H: 1,000\end{array}$ & & $\begin{array}{l}A: 1,000 \\
B: 1,000\end{array}$ & $\begin{array}{l}A: 1,000 \\
B: 1,000\end{array}$ & $\begin{array}{l}A: 1,000 \\
B: 1,000\end{array}$ \\
\hline 1998 & $\begin{array}{l}M: 1,000 \\
H: 1,000\end{array}$ & $\begin{array}{l}M: 1,000 \\
H: 0,022\end{array}$ & $\begin{array}{l}M: 1,000 \\
H: 0,601\end{array}$ & $\begin{array}{l}M: 1,000 \\
H: 1,000\end{array}$ & & $\begin{array}{l}A: 1,000 \\
B: 1,000\end{array}$ & $\begin{array}{l}A: 1,000 \\
B: 1,000\end{array}$ \\
\hline 1999 & $\begin{array}{l}M: 1,000 \\
H: 1,000\end{array}$ & $\begin{array}{l}M: 1,000 \\
H: 0,081\end{array}$ & $\begin{array}{l}M: 0,113 \\
H: 1,000\end{array}$ & $\begin{array}{l}M: 1,000 \\
H: 1,000\end{array}$ & $\begin{array}{l}M: 1,000 \\
H: 1,000\end{array}$ & & $\begin{array}{l}A: 1,000 \\
B: 1,000\end{array}$ \\
\hline 2000 & $\begin{array}{l}M: 1,000 \\
H: 1,000\end{array}$ & $\begin{array}{l}M: 0,011 \\
H: 0,009\end{array}$ & $\begin{array}{l}M: 0,000 \\
H: 0,278\end{array}$ & $\begin{array}{l}M: 1,000 \\
H: 1,000\end{array}$ & $\begin{array}{l}M: 1,000 \\
H: 1,000\end{array}$ & $\begin{array}{l}M: 1,000 \\
H: 1,000\end{array}$ & \\
\hline
\end{tabular}

\section{Tableau III}

Résultats de l'Analyse de Variance à deux facteurs de variabilité (Année et Milieu) sur les paramètres des seuls échantillons réalisés dans les zones moyenne et basse du réservoir de Petit-Saut.

Table III

Results of two way analysis of variance with year and habitat on parameter of the samples taken in medium and lower sections of Petit-Saut reservoir.

\begin{tabular}{|l|r|c|c|}
\hline Facteurs & Nombre & Diversité & Equitabilité \\
\hline Année & $<0,001$ & 0,005 & $<0,001$ \\
\hline Milieu & 0,103 & 0,104 & 0,281 \\
\hline Année ${ }^{\star}$ Milieu & 0,945 & 0,052 & 0,062 \\
\hline
\end{tabular}



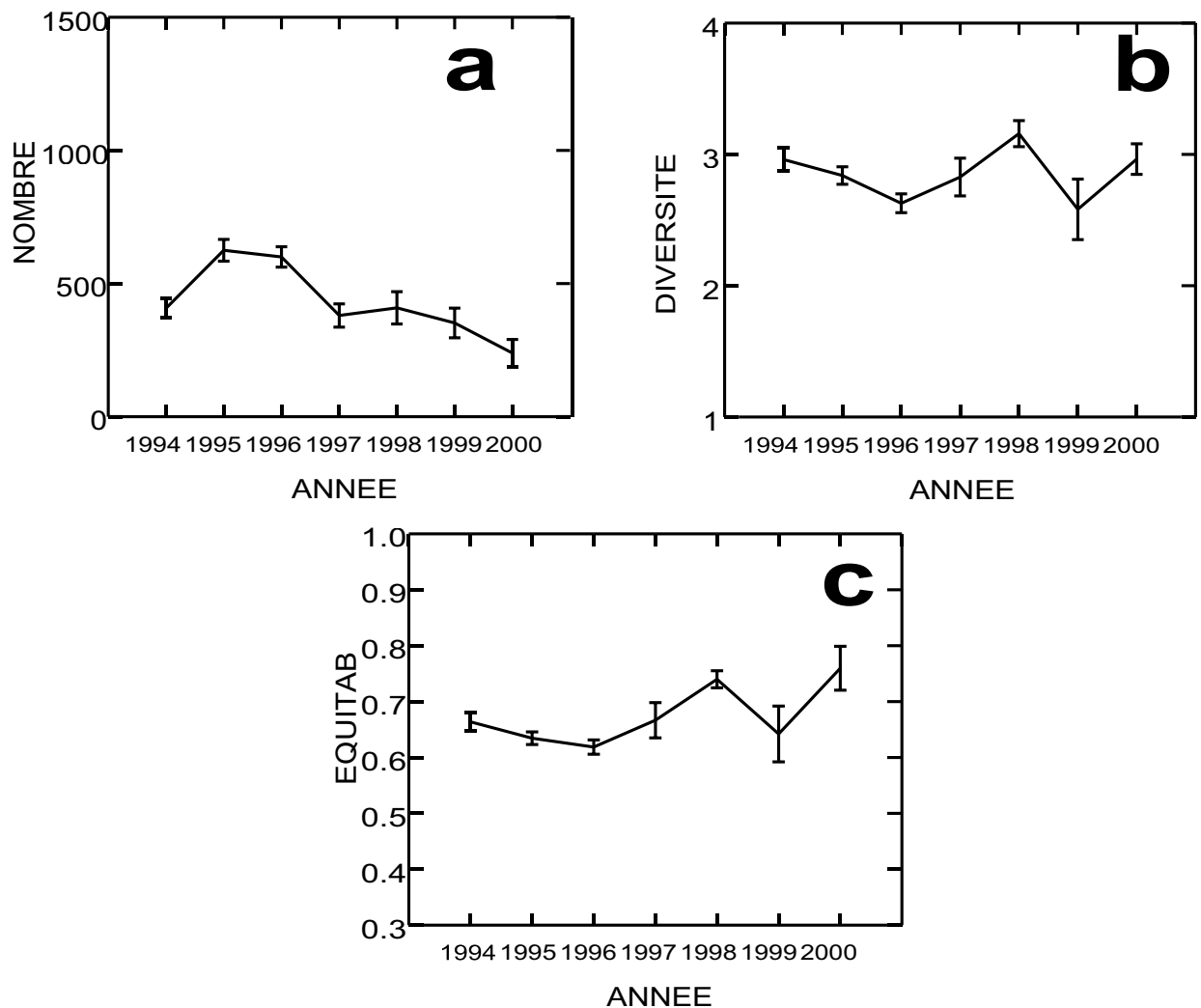

Figure 5

Variations significatives des paramètres des échantillons dans les zones moyenne et basse de la retenue en fonction des facteurs année et milieu. $a$ : nombre capturé en fonction de l'année. b : diversité en fonction de l'année. c : equitabilité en fonction de l'année. Les barres d'erreurs correspondent à une erreur type.

Figure 5

Significant changes in samples parameters in medium and low sections of the reservoir with year and habitat. a: captured number with year. b: diversity with year. c: equitability with year. Error bars correspond to one standard error.

\section{Tableau IV}

Résultat des ajustements du nombre cumulé d'espèces dans les différentes périodes à un modèle logistique (voir le texte pour les détails du modèle).

Table IV

Results of non linear adjustments of cumulated number of species to a logistic model (see text for detail of the model).

\begin{tabular}{|l|r|c|c|}
\hline Facteurs & Nombre & Diversité & Equitabilité \\
\hline Année & $<0,001$ & 0,005 & $<0,001$ \\
\hline Milieu & 0,103 & 0,104 & 0,281 \\
\hline Année*Milieu & 0,945 & 0,052 & 0,062 \\
\hline
\end{tabular}




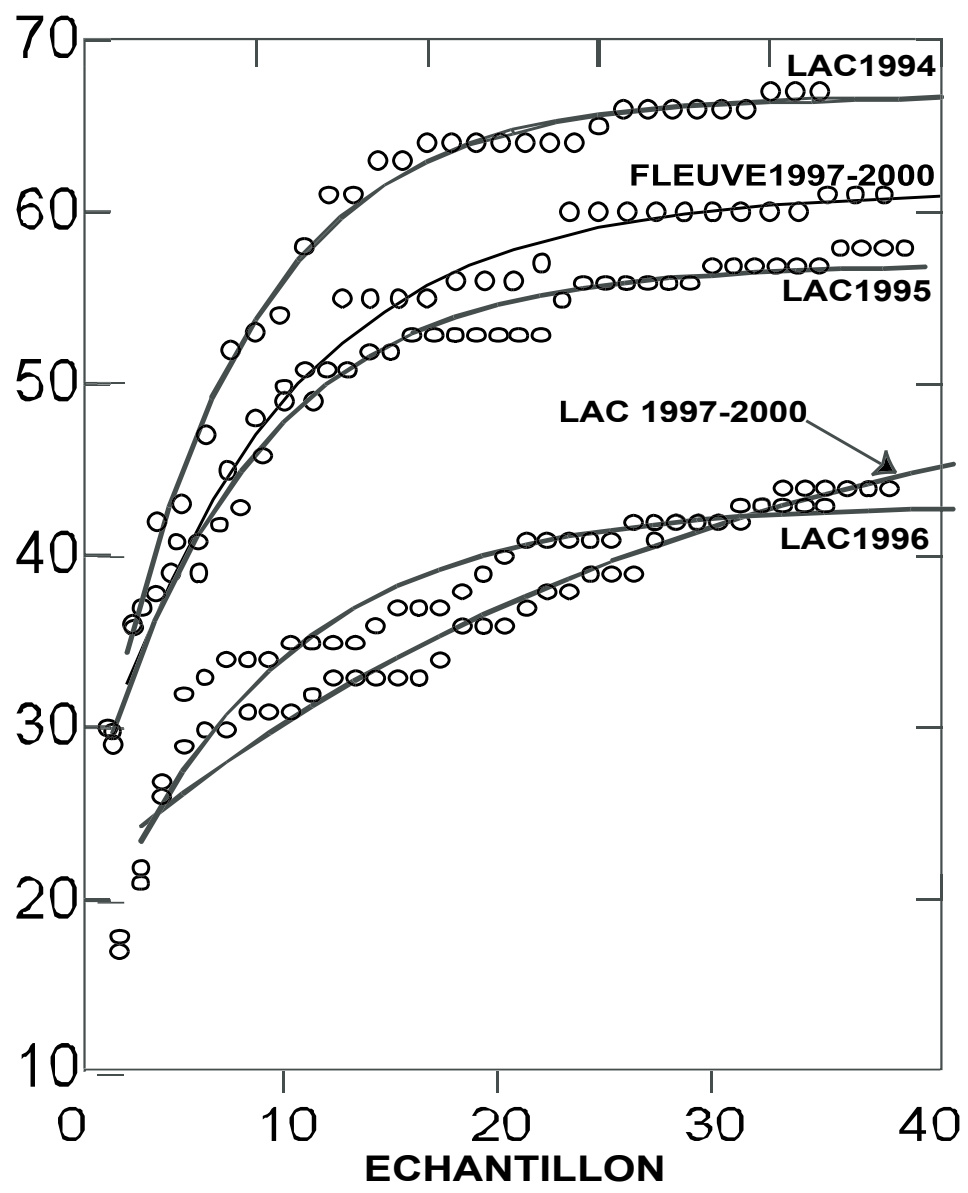

Figure 6

Nombre d'espèces cumulé au cours des années 1994, 1995, 1996 et de la période 1997 à 2000.

\section{Figure 6}

Cumulated number of species during the years 1994, 1995, 1996 and the period 1997 to 2000.

\section{Composition spécifique}

L'analyse des correspondances effectuée sur les prélèvements de juin et novembre depuis 1994 et jusqu'en 2000 fait apparaître une transformation rapide des peuplements du réservoir au cours de la première année d'inondation (Figure 7). L'évolution est plus lente au cours des deux années suivantes et il semble y avoir une certaine stabilisation à partir de la quatrième année de mise en eau. Les échantillons de la tête de la retenue (front) sont intermédiaires entre ceux du réservoir et ceux du fleuve à l'amont et ni le front, ni le fleuve, ne montrent d'évolution interannuelle continue au cours des 7 années d'observation en ce qui concerne la composition spécifique de leur peuplement.

La première année d'existence du réservoir était marquée par la particulière abondance de Parauchenipterus galeatus (Linnaeus, 1766), une espèce absente du fleuve en amont. Cette population a décliné rapidement dès la fin de la première année pour se stabiliser à un niveau très bas (Figure 8 ). 


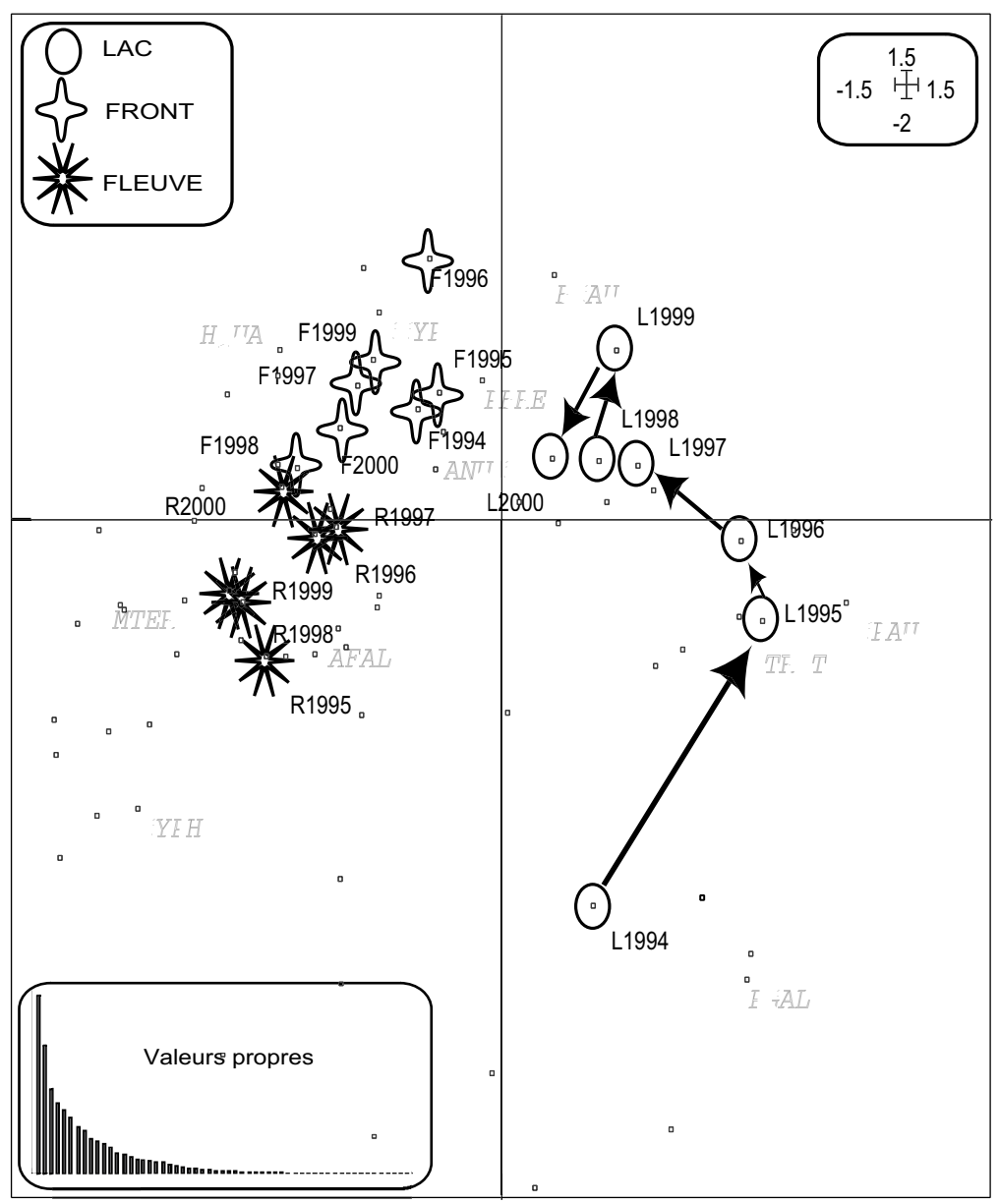

Figure 7

Projection des échantillons et des espèces sur les deux premiers axes de l'analyse des correspondances. Axe 1 horizontal (18\%) ; axe 2 vertical (13\%). Les symboles représentent les centres de gravité des points correspondant à la position indiquée par la première lettre $(R=$ rivière ; $F=$ front $; L=$ lac) pour l'année indiquée à la suite.

AFAL $=$ Acestrorhynchus falcatus. ANUC $=$ Auchenipterus nuchalis.

BCAU = Bryconops caudomaculatus. CCYP = Curimata cyprinoides.

CPAU = Charax pauciradiatus. $\mathrm{CYPH}=$ Cyphocharax spp.

HQUA = Hemiodopsis quadrimaculatus. MTER = Myleus ternetzi.

PGAL $=$ Parauchenipterus galeatus. PBRE $=$ Poptella brevispina.

TROT = Triportheus rotundatus.

Figure 7

Projection of samples and species on the two first factors of correspondance analysis. Factor 1 horizontal (18\%); factor 2 vertical (13\%). Symbols are the centroids of each position/year set of data. The first letter shows the position $(R=$ river; $F=$ head of reservoir; $L=$ reservoir) and the following number is the year. AFAL = Acestrorhynchus falcatus. ANUC = Auchenipterus nuchalis.

BCAU = Bryconops caudomaculatus. CCYP = Curimata cyprinoides .

CPAU = Charax pauciradiatus. $\mathrm{CYPH}=$ Cyphocharax spp.

HQUA = Hemiodopsis quadrimaculatus. MTER = Myleus ternetzi.

PGAL $=$ Parauchenipterus galeatus. PBRE $=$ Poptella brevispina.

TROT = Triportheus rotundatus. 

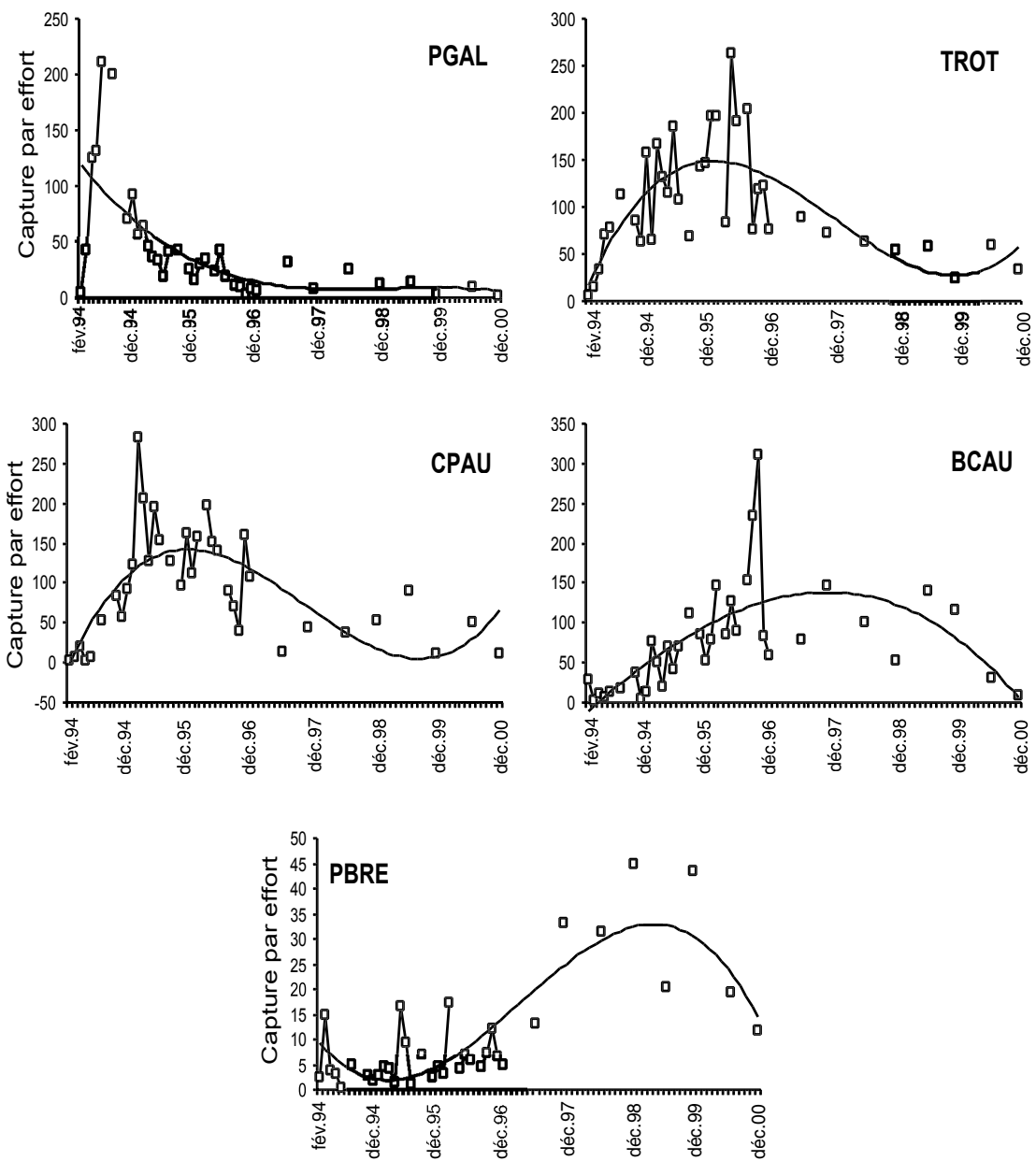

Figure 8

Variations temporelles de la capture des principales espèces dans la retenue de Petit-Saut. Les courbes représentent un ajustement polynomial d'ordre 3 . PGAL $=$ Parauchenipterus galeatus . TROT $=$ Triportheus rotundatus.

CPAU = Charax pauciradiatus $. \mathrm{BCAU}=$ Bryconops caudomaculatus .

PBRE $=$ Poptella brevispina .

\section{Figure 8}

Temporal changes of catches for the main species in Petit-Saut reservoir. The curves are polynomial adjustment of $3^{\text {rd }}$ order.

PGAL $=$ Parauchenipterus galeatus . TROT $=$ Triportheus rotundatus.

CPAU = Charax pauciradiatus. $\mathrm{BCAU}=$ Bryconops caudomaculatus.

PBRE = Poptella brevispina.

Deux espèces étaient les plus déterminantes pour la définition des peuplements des deux années suivantes: Triportheus rotundatus (Schomburgk, 1841) et Charax pauciradiatus (Günther, 1864). Les populations de ces deux espèces ont été dominantes pendant la deuxième et troisième année et se sont maintenues à partir de 1997 à un niveau moyen (Figure 8). Elles ont été progressivement substituées par Bryconops caudomaculatus (Günther, 1869) dont la population atteignait un maximum vers la fin de l'année 1996, puis par Poptella brevispina (Reis, 1989), à partir de la fin 1997 (Figure 8). L'année 2000 a vu une diminution générale des captures de ces espèces. 


\section{DISCUSSION}

On distingue classiquement 3 phases dans l'histoire d'une retenue : la phase de remplissage, la phase de stabilisation physique et la phase de stabilisation écologique (SCOPE WORKING GROUP ON MAN-MADE LAKES, 1972). La première consiste en une montée plus ou moins régulière de l'eau qui inonde progressivement la végétation. Le milieu est alors extrêmement dynamique, et un énorme espace est soudainement disponible pour les poissons. La deuxième voit l'accélération des processus de décomposition de la matière organique noyée avec pour conséquence la libération de grandes quantités de nutriments mais aussi l'émission de composés toxiques et une désoxygénation généralisée. Enfin, une phase de stabilisation écologique constitue l'installation lente d'un certain équilibre entre production primaire et production terminale.

La retenue de Petit-Saut a été remplie en deux temps : de janvier à juillet 94 pour atteindre très rapidement le niveau $31 \mathrm{~m}$ puis de décembre 94 à mai 95 jusqu'au niveau $35 \mathrm{~m}$. En dépit de la désoxygénation généralisée due aux processus de décomposition de l'énorme quantité de matière organique de la forêt inondée, les poissons ont pu se développer dès les premiers mois dans la fine couche oxygénée en surface. Les captures étaient élevées et suivaient une courbe ascendante. Les différentes zones du lac étaient relativement homogènes. La richesse spécifique était élevée car toutes les espèces n'avaient d'autre choix que de se trouver dans la couche oxygénée, seul milieu habitable, et étaient ainsi vulnérables à la capture.

Une espèce a profité rapidement de ces conditions : Parauchenipterus galeatus. II s'agit d'un poisson très robuste dont la distribution avant barrage était limitée à la partie basse du fleuve (TITO DE MORAIS et LAUZANNE, 1994). L'espèce présente une saison de reproduction s'étendant durant toute la durée de la saison des pluies de décembre à août (PONTON et MERONA, 1998). Son régime alimentaire est omnivore opportuniste et, à cette époque, il consommait en grandes quantités le matériel végétal de la forêt ennoyée ainsi que des insectes terrestres (obs. pers. non publiées). C'est aussi une espèce bien adaptée aux milieux hypoxiques, préférant les eaux calmes et les marais plutôt que les zones courantes (LE BAIL et al., 2000). Les spécimens récoltés en mars ont une taille moyenne de $90 \mathrm{~mm}$ et en avril la structure en taille comporte un mode marqué à $130 \mathrm{~mm}$ qui croit en mai jusqu'à $150 \mathrm{~mm}$ (MERONA, 2001). Une croissance précoce aussi rapide serait compatible avec les observations de MEUNIER et al. (1994) sur d'autres Siluriformes en Guyane. Ainsi l'abondante population de $P$. galeatus pourrait bien être issue d'une reproduction ayant eu lieu au tout début de la saison des pluies 1993-1994 suivie d'une survie juvénile exceptionnelle et d'une croissance favorisée par l'abondance de la nourriture disponible. La rapide raréfaction des ressources alimentaires avec la mort de la végétation noyée n'a pas permis à cette espèce de se maintenir et son abondance a rapidement décliné dès la fin de la phase de remplissage.

La deuxième phase dans l'évolution de la retenue de Petit-Saut commence dès la fin du remplissage. La plus grande part de la surface de la retenue était déjà en eau et le niveau montait plus lentement jusqu'à sa cote maximum atteinte mi-1995. On a assisté alors à une augmentation progressive de la couche d'eau oxygénée en surface et à un développement explosif de plancton (RICHARD et al., 1997 ; HOREAU et al., 1997). Cette phase s'est poursuivie jusqu'à fin 1996 soit 3 ans après la fermeture du barrage. Les captures de poissons restaient élevées mais la zone basse se différenciait de plus en plus de la zone moyenne avec une densité plus faible de poissons. La richesse spécifique diminuait sensiblement avec un déficit de plus de 20 espèces par rapport à la première année. Cette période était caractérisée par la particulière abondance de deux espèces. L'une, Triportheus rotundatus est un Characidae pélagique de surface dont le régime alimentaire est, dans les conditions fluviales, composé de végétaux et d'insectes terrestres. L'espèce a du bénéficier d'un recrutement extrêmement favorable lors de la phase d'inondation. Les jeunes ont pu trouver, dans les milieux nouvellement inondés, de la 
nourriture en abondance et une certaine protection contre les prédateurs. De plus, dans le lac, l'espèce a tiré parti du développement du plancton en consommant de grandes quantités de copépodes (observations non publiées). Les mêmes considérations peuvent être avancées pour ce qui concerne la deuxième espèce : Charax pauciradiatus, Characidae de plus petite taille qui consommait dans le lac plutôt des larves de Chaoborus.

Enfin, la dernière phase dans la série d'observations était caractérisée par une diminution lente et progressive des captures, une augmentation parallèle de la richesse spécifique et la suprématie de deux espèces : Bryconops caudomaculatus et Poptella brevispina. Ces deux espèces ont des régimes alimentaires voisins de ceux de $T$. rotundatus et leur suprématie aux dépens de cette dernière est très probablement due à une stratégie vitale différente plus adaptée au nouveau milieu. Les deux espèces sont de plus petite taille et leur reproduction est continue (PONTON et MERONA, 1998). Cette période a été marquée par deux événements hydrologiques exceptionnels : d'une part, une saison hydrologique très déficitaire en 1998 qui a conduit à un abaissement du niveau du lac jusqu'à sa cote minimum et d'autre part une crue extrêmement importante en 2000. A partir de 1997, l'oxycline s'est abaissée amenant des concentrations en oxygène de l'ordre de 2 mg. $\mathrm{I}^{-1}$ jusqu'à des profondeurs de plus de $4 \mathrm{~m}$ (RICHARD et al., 2000). L'augmentation du volume ainsi disponible pour les poissons peut expliquer en partie la baisse observée dans la capture par un phénomène de dilution. Des essais d'utilisation de filets de $4 \mathrm{~m}$ de chute ont cependant montré que le volume des captures diminuait rapidement avec la profondeur. La sécheresse exceptionnelle de 1998 a affecté seulement le fleuve à l'amont et la partie haute du lac sous l'influence directe du fleuve. Les deux espèces qui dominaient les échantillons à cette époque étaient toutes deux des petits Characidae en majorité insectivores.

II est clair à l'examen des graphiques que la retenue n'est pas parvenue à un état de relatif équilibre après 7 ans. Les captures suivent toujours une courbe descendante et la composition spécifique des échantillons évolue constamment.

Bien que les séries d'observations continues soient rares dans les situations de barrages en zone tropicale, il apparaît que le schéma observé dans le cas de la retenue de Petit-Saut est conforme aux évolutions constatées ailleurs.

Le développement explosif d'une population pendant la phase de remplissage a été rapporté en Afrique pour les lacs Kariba et Kainji (KAPETSKY et PETR, 1984) et en Amérique du Sud pour les lacs d'Itaipu et de Tucurui (AGOSTINHO et al., 1999 ; LEITE, 1993 ; SANTOS et MERONA, 1996). II est d'ailleurs intéressant de constater que, dans ce dernier cas, la même espèce qu'à Petit-Saut (Parauchenipterus galeatus) a eu un comportement identique lors du remplissage de la retenue de Tucurui sur le fleuve Tocantins.

A Itaipu AGOSTINHO et al. (1999) considèrent une phase unique, qu'ils appellent phase de colonisation, pour les 15 années d'observations qui ont suivi le remplissage de la retenue. Dans cette retenue il apparaît en effet que les captures par effort suivent des évolutions monotones pendant toute la période suivant le remplissage, déclinantes dans les zones lacustres et riverines et relativement constantes dans la zone de transition. En revanche, ce pic de production a souvent été signalé dans les grandes retenues intertropicales et a donné lieu à l'installation de pêcheries importantes (BALON et COCHE, 1974 ; PETR, 1975 ; KAPETSKY et PETR, 1984). Cette production élevée est clairement due à la profusion de ressources allochtones provenant de la décomposition du matériel végétal inondé (BAXTER, 1977 ; PETR, 1978). Sa durée et son intensité sont étroitement dépendantes de la zone climatique, du type de végétation, de la durée du remplissage (PETR, 1975). Le type d'espèces qui contribuent le plus à cette production élevée est très variable d'une situation à l'autre. Pour qu'une population se développe d'une manière explosive dans les premières années d'implantation d'une retenue, il faut d'une part que 
sa période de reproduction ait coïncidé avec le début du remplissage et que sa durée de vie soit compatible avec la durée des processus de décomposition. II faut d'autre part qu'elle puisse tirer parti des abondantes ressources allochtones disponibles dans le lac nouvellement formé.

La période suivante dans l'histoire d'une retenue consiste en une diminution lente et continue de la production lorsque le système passe progressivement d'un fonctionnement hétérotrophe à un fonctionnement autotrophe (BAXTER, 1977). Cette période peut s'étendre sur de longues années. Dans le lac Kariba, mis en eau en 1958, les captures par effort d'une pêcherie expérimentale ont décliné jusqu'en 1973 soit 15 ans après la fermeture (MACHENA, 1995). Dans le lac Kainji les rendements de la pêche commerciale ont diminué pendant les 7 années suivant la fermeture puis semblaient se stabiliser (ITA, 1984). Enfin dans la retenue d'Itaipu, AGOSTINHO et al., 1999 estiment que l'équilibre n'est pas encore atteint 15 ans après la fermeture au vu des captures par effort encore déclinantes au moins dans la partie lacustre.

\section{CONCLUSIONS}

Le barrage de Petit-Saut constitue une situation d'étude privilégiée pour le suivi sur le long terme des effets d'un barrage hydroélectrique implanté en zone de grande forêt équatoriale. Jusqu'à l'année 2000 , un comité scientifique a fonctionné à l'initiative d'EDF en émettant des recommandations sur la nécessité de contrôler les différents compartiments de l'environnement. Un programme de suivi minimum a été mis en place et il semble que EDF ait la volonté de le continuer jusqu'à l'atteinte d'un équilibre. Ce programme de suivi couvre un large champ d'investigations et génère des données sur la dégradation de la matière organique et le cycle du méthane, sur la qualité physicochimique de l'eau, sur la dynamique des invertébrés et des poissons et sur l'accumulation de mercure chez les poissons. Ainsi la situation de Petit-Saut constituera le tout premier ensemble de données homogènes permettant de visualiser les effets à long terme d'un barrage en zone tropicale forestière.

\section{REMERCIEMENTS}

Ce travail a été rendu possible grâce à l'ardeur et l'enthousiasme de l'équipe du laboratoire d'hydrobiologie du Centre IRD de Cayenne: Jean-Claude BRON, Rolland RUFFINE et Michel TARCY. Je voudrais aussi remercier toute l'équipe du laboratoire Hydreco de Petit-Saut pour l'appui qu'elle nous a toujours apporté avec la meilleure bonne volonté. Les études ont été intégralement financées par EDF.

\section{BIBLIOGRAPHIE}

ACKERMANN W.C., WHITE G.F., WORTHINGTON E.B. (Eds.), 1973. Man-Made Lakes : Their Problems and Environmental Effects. American Geophysical Union, 847 p.

AGOSTINHO A.A., MIRANDA L.E., BINI L.M., GOMES L.C., THOMAZ S.M., SUZUKI H.I., 1999. Patterns of Colonization in Neotropical Reservoirs, and Prognoses on Aging. In : TUNDISI J.G. and STRASKRABA M. (Eds.), Theoretical Reservoir Ecology and its Applications, International Institute of Ecology, Brazilian Academy of Sciences and Backhuys publishers, 227-265.

BALON E.K., COCHE A.G. (Eds.), 1974. Lake Kariba : A Man-Made Tropical Ecosystem in Central Africa., Dr. W. Junk b.v. The Hague, 767 p.

BAXTER R.M., 1977. Environmental effects of dams and impoundments. Ann. Rev. Ecol. Syst., 8, 255-283. 
HOREAU V., CERDAN P., CHAMPEAU A., 1997. La mise en eau du barrage hydroélectrique de Petit-Saut (Guyane) : ses conséquences sur les peuplements d'invertébrés aquatiques et sur la nourriture des poissons. Hydroécol. Appl., 9 (1-2), 213-240.

ITA E.O., 1984. Kainji (Nigeria). In : KAPETSKY J.M. and PETR T. (Eds./Rev.), Status of African reservoir fisheries / Etat des pêcheries dans les réservoirs d'Afrique. FAO, CIFA Technical Paper, 43, $104 \mathrm{p}$.

KAPETSKY J.M., PETR T., 1984. Status of African reservoir fisheries / Etat des pêcheries dans les réservoirs d'Afrique. FAO, CIFA Technical Paper, 10, $326 \mathrm{p}$.

LE BAIL P.Y., KEITH P., PLANQUETTE P., 2000. Atlas des poissons d'eau douce de Guyane. Tome 2, fasc. 2. M.N.H.N., S.P.N., I.E.B.G., 307 p.

LEITE R.A.N., 1993. Efeitos da usina hidroelétrica de Tucuruí sobre a composição da ictiofauna das pescarias experimentais de malhadeiras realizadas no baixo Tocantins (Pará). Diss. Mestrado, INPA/FUA, Manaus (Am), 133 p.

LIND O.T., TERRELL T.T., KIMMEL B.L., 1993. Problems in reservoir trophic-state classification and implications for reservoir management. In : STRASKRABA M., TUNDISI J.G. and DUNCAN A. (Eds.), Comparative Reservoir Limnology and Water Quality Management, Kluwer Publishers, 57-67.

LOWE-MCCONNELL R.H. (ed.), 1966. Man-Made Lakes. Academic Press, 218 p.

MACHENA C., 1995. Recent developments in the fisheries of Lake Kariba (Zambia / Zimbabwe). In : CRUL R.C.M. and ROEST F.C. (Eds.), Current status of the fisheries and fish stocks of the four largest African reservoirs : Kainji, Kariba, Nasser / Nubia and Volta, FAO, CIFA Technical Paper, 41-80.

MERONA B. de, 2001. Aménagement hydroélectrique de Petit-Saut. Etudes relatives au suivi ichtyologique dans la retenue et à l'aval. Synthèse des études. IRD Cayenne, 76 p.

MEUNIER F.J., ROJAS-BELTRAN R., BOUJARD T., LECOMTE F., 1994. Rythmes saisonniers de la croissance chez quelques Téléostéens de Guyane française. Rev. Hydrobio. trop., 27 (4), 423-440.

OBENG L.E. (ed.), 1969. Man-Made Lakes: The Accra Symposium, Ghana Universities Press, $398 \mathrm{p}$.

PETR T., 1975. On some factors associated with the initial high fish catches in new African man-made lakes. Arch.Hydrobiol., 75 (1), 32-49.

PETR T., 1978. Tropical man-made lakes - their ecological impact. Arch. Hydrobiol., 81 (3), 368-385.

PONTON D., MERONA B. de, 1998. Fish life-history tactics in a neotropical river with a highly stochastic hydrological regime: the Sinnamary River, French Guiana, South America. Pol. Arch. Hydrobiol., 45 (2), 201-224.

RICHARD S., ARNOUX A., CERDAN P., 1997. Evolution de la qualité physico-chimique des eaux de la retenue et du tronçon aval depuis le début de la mise en eau du barrage de Petit-Saut. Hydroécol. Appl., 9 (1-2), 57-84.

RICHARD S., GALY-LACAUX C., ARNOUX A., CERDAN P., DELMAS R., DUMESTRE J.F., GOSSE P., HOREAU V., LABROUE L., SISSAKIAN C., 2000. Evolution of physicochemical water quality and methane emissions in the tropical hydroelectric reservoir of Petit-Saut (French Guiana). Verh. Internat. Verein. Limnol., 27, 1454-1458.

SCOPE WORKING GROUP ON MAN-MADE LAKES, 1972. Man-Made Lakes as modified ecosystems. International Council of Scientific Unions, $76 \mathrm{p}$.

SANTOS G.M. dos, MERONA B. de, 1996. Impactos imediatos da UHE Tucurui sobre as comunidades de peixes e a pesca. In : MAGALHAES S.B., BRITTO R.C., CASTRO E.R. de (Org.). Energia na Amazônia. Vol. I, 251-258, Museu Paraense Emílio Goeldi, UFPA, Assoc. Univ. Amazônicos.

STRASKRABA M., TUNDISI J.G., DUNCAN A. (Eds.), 1993. Comparative Reservoir Limnology and Water Quality Management., Kluwer Academic Publishers, $291 \mathrm{p}$.

THIOULOUSE J., CHESSEL D., DOLEDEC S., OLIVIER J.M., 1997. ADE-4 : a multivariate analysis and graphical display software. Statistics and Computing, 7, 75-83. 
THORNTON J.A., RAST W., 1993. A test of hypotheses relating to the comparative limnology and assessment of eutrophication in semi-arid man-made lakes. In : STRASKRABA M., TUNDISI J.G. and DUNCAN A. (Eds.), Comparative Reservoir Limnology and Water Quality Management, Kluwer Publishers, 1-24.

TITO DE MORAIS L., LAUZANNE L., 1994. Zonation longitudinale des peuplements ichtyques avant mise en eau de la retenue de Petit-Saut (Guyane française). Rev. Hydrobiol. Trop., 27 (4), 467-484.

TUNDISI J.G., STRASKRABA M. (Eds.), 1999. Theoretical Reservoir Ecology and its Applications, Backhuys Publishers, $583 \mathrm{p}$.

VAN DER HEIDE J., 1982. Lake Brokopondo. Filling phase limnology of a man-made lake in the humid tropics., Offserdrukkerij Kanters b.v., 427 p. 